\title{
Preliminary design of a tendon-based anthropomorphic robotic hand
}

\author{
S. Oung ${ }^{1}$, B. M. Pohl ${ }^{1,2}$, U. G. Hofmann ${ }^{1,3}$ \\ IInstitute for Signal Processing, University of Lübeck, Lübeck, Germany, hofmann@isip.uni-luebeck.de \\ ${ }^{2}$ Graduate School for Computing in Medicine and Life Sciences, Lübeck, Germany \\ ${ }^{3}$ Research Group for Neuroelectronic Systems, University Medical Center Freiburg, Freiburg Germany
}

\begin{abstract}
With the development of more advanced technology, neuroprosthetics are becoming a more viable solution for amputees. However, a limitation on designing neural-machine interfaces is the lack of robotic hand availability in academic institutes. Although commercial hands exists and have the aptitude of mimicking the human hand, such systems are large and costly. The Lübeck's anthropomorphically designed robotic hand (LAnDRoH) is meant to address these issues by providing a lightweight and cost-effective solution with the capabilities of a fully dexterous hand. The LAnDRoH is an endoskeletal robotic hand that is designed to mimic the appearance and kinematics of an adult human hand. It was constructed with a polyamide polymer and uses 25 actuators to control 20 degrees of freedom. A flexible link-hosted actuation system, using braided Dyneema ${ }^{\circledR}$ cables in a $\mathrm{N}+1$ routing configuration, mimics the functionality of tendons in the human hand. This paper presents the design and preliminary tests of the LAnDRoH.
\end{abstract}

\section{Introduction}

Within the past year, an estimated of 158,000 individuals underwent amputation with an expectation that the overall number of amputations is increasing [1]. Amputation, whether it is the result of congenital or medical deficiencies, leads to a drastic restriction in function and cosmesis. Numerous prosthetics have been developed in attempt to restore these attributes. However, these prosthetics are still limited in function. Research has shown that $30-50 \%$ amputees reject the use of their prosthetic hands due to heavy weight and low functionality [2]. The field of neuroprosthetics is hoping to address this by providing a higher functional robotic replacement that can be manipulated through an active nervous control interface.

Prosthetic hands are of particular interest for researchers, due to its mechanical and control complexity [3]. Extensive amount of research in trans-radial robotic manipulation has lead to a variety of designs. Some research groups have focused on developing a fully dexterous hand capable of accurately approximating both the manipulation and grasping ability of the human hand. Dexterous hands such as the DLR Hand [4], NASA's Robonaut Hand [5], as well as commercially available robotic hands, such as the Shadow Hand (Shadow Robot Company, UK), have already been developed. These types of hands are capable of performing power and precision grips to a high degree of accuracy. However, they are generally large and costly to acquire due to the required complex control and mechanical systems.

Other groups have focused on developing a completely self-contained robotic hand within the volume size of a human hand. This development is directed for immediate prosthetic use. However, they are normally underactuated due space limitations [3]. Several joints are coupled with passive mechanisms, which leads to an unnatural movements and renders the prostheses cosmetically and functionally unappealing [6].

A lot of effort has been done worldwide to overcome these disadvantages. In this paper, the design and preliminary tests of a new robotic hand is presented. The Lübeck's anthropomorphically designed robotic hand (LAnDRoH) is based on developing a cost-effective solution that has a similar range of motion as the human hand and can be fully articulated. This robot is designed to serve as a research tool to help advance humanoidrobotic interfaces such as brain-machine and natural interfaces. To this end, the full system was not limited to a self-contained system within the trans-radial region.

\section{Methods}

\subsection{Kinematic Design}

The following criterions were used for designing the LAnDRoH.

\section{Design Criterion}

- The complete structure should appear similar to an adult hand with 5 fingers

- Size and weight should resemble the average naked human hand

- The range of finger movement should realize practical functions such as opening to clenching bulk objects - As far as the first three conditions are satisfied, the system cost should be kept at a minimum

- As far as the fourth condition is satisfied, the speed of the system should resemble that of the human hand, with a force output at each finger tip of $25 \mathrm{~N}$ with straight posture. 
This work was based on the appearance and kinematics of the $50^{\text {th }}$ percentile adult male hand. It is composed of 20 links that imitate the four fingers and thumb. The carpal and metacarpal bones of the fingers were compressed to form the LAnDRoH's baseplate.

The model consists of 20 degrees of freedom (DOF), which represent the active joints within the hand. For this preliminary design, a single kinematic configuration was considered. Subsequently, the thumb and fingers function identically with just a difference in angle and offset with respect to the baseplate. The configuration of each finger is defined by serial set of 4 single DOFs joints, connected by 4 links, shown in Figure 1. In this configuration, the proximal (PIP) and distal (DIP) interphalangeal joints are modelled with a single DOF joint, which allows for the flexion and extension of the joints. Conversely, the metacarpophalangeal joint (MCP) is modelled by two perpendicular single DOF joints; one for flexion/ extension, and the other for abduction/adduction. The main difference with this model is the separation of the natural 2 DOF MCP joint into 2 single active joints. The abduction/adduction of the MCP is defined before the flexion/extension to better simulate finger displacements.

The range of motion of each joint mimics the human hand, shown in Figure 2. Each finger also is capable of $15^{\circ}$ of abduction and adduction respectively.

\subsection{Force Transmission}

The LAnDRoH is a fully actuated system that allows independent control of each link. The control is based on a link-hosted actuation system. Flexible links were made from braided Dyneema ${ }^{\circledR}$ polymer to transmit the force from the actuators to the fingers, similar to the natural tendons. These artificial tendons were organised in a $\mathrm{N}+1$ configuration where $\mathrm{N}$ represents the number of joints. This routing configuration allowed for complete actuation of each link, similar to the standard $2 \mathrm{~N}$, antagonistagonistic, configuration. However, the main advantage of this configuration is a reduced number of required actuators, and subsequently smaller actuation package [7]. The current configuration uses an agonistic tendon to flex each joint, and a single antagonistic tendon to extend the finger. Minimal pretension was applied to the tendons to remove any dead-space in the tendons that would lead to reduced responsiveness.

In this initial design, the force was generated by $\mathrm{S} 125$ sail winch (GWS, Taiwan) servo motors attached to $\varnothing 40 \mathrm{~mm}$ capstans.

\subsection{Mechanical Design}

The LAnDRoH is an endoskeletal frame constructed of PA2200 (EOS e-Manufacturing Solutions, Germany), a polyamide powder that was sintered using a rapid prototype machine. A rubber based synthetic skin is intended to cover the frame to provide cosmesis as well as frictional grip when grasping objects.

The general shape of each link deviated from the rigid cylindrical or rectangular shape and used a more hyperbolic form. This served the primary purpose of allowing straight connections between contact points for the tendons. This avoided unnecessary wrapping around the links. This would have lead to an increase in internal friction as well as additional points of contact where force is applied on the link.

As opposed to an enclosed pin-hinge system that is commonly seen in single DOF designs, an open interlocking joint structure was implemented. In Figure 1,

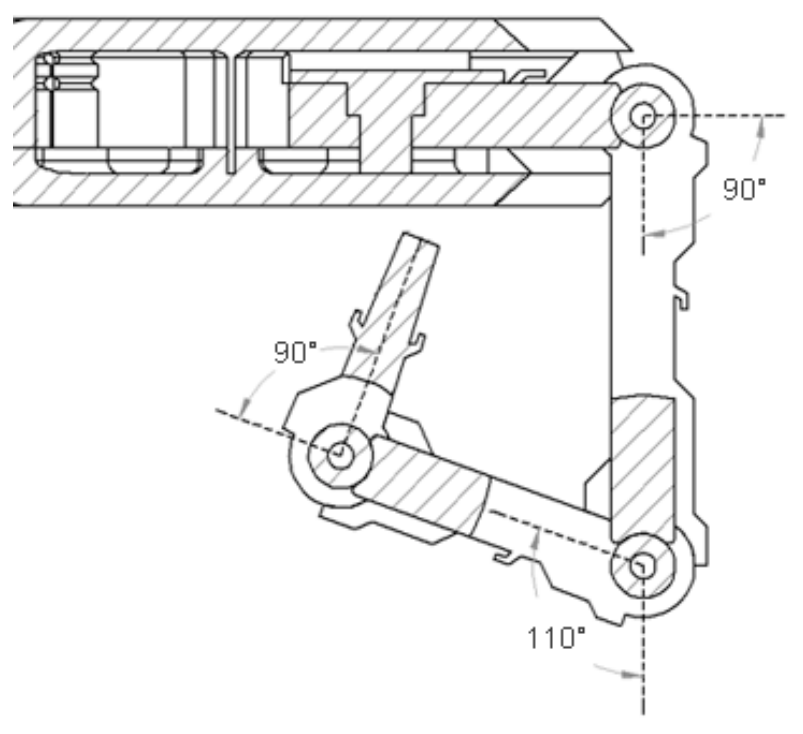

Figure 2 LAnDRoH's maximum range of motion

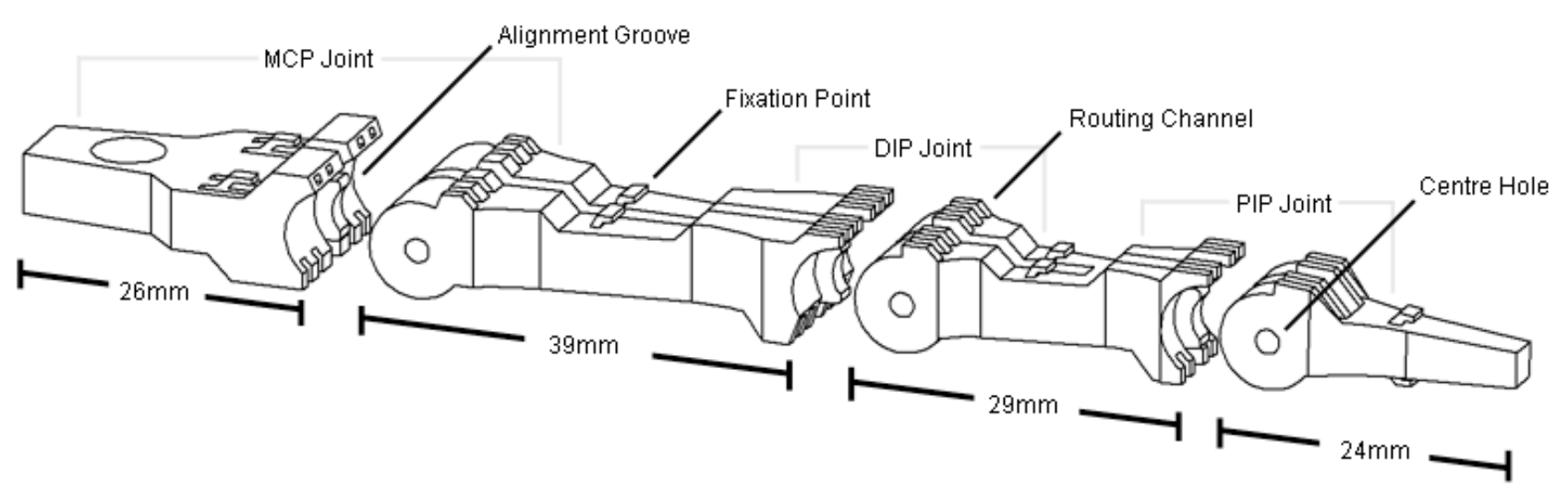

Figure 1 An exploded view of the mechanical design of a LAnDRoH's finger 
adjacent links were held through the tension in the tendons, and are aligned through with an alignment groove. This open joint connection allowed for a more robust system. Applied lateral forces would primarily be transmitted to the tendons. However, if large impact force is exerted, the link will simply dislocate without any overall damage to the endoskeletal frame. Once the applied force is released, the links would return to their original position with the help of the alignment groove.

\subsection{Finger Control}

For effective control of the joint structures, it was necessary to derive a mathematical model to relate the contraction and extension of the tendons with the joint angles. As aforementioned, maintaining tension within the tendons is a high priority to ensure connection between the joints.

Two mathematical models were created to describe the relationship between the change in length of the tendon with respect to the joint angles. The two equations modelled the possibilities of the tendon wrapping around the frontal and dorsal portion of the joints. The implemented models used the assumption of inelastic tendons. This was justified by the fact that the maximum change of length due to maximum flexion was within the range of millimetres, and added that the rated elongation before break is maximum 4\% [8]. Any added accuracy of an elastic model could not be reflected by the resolution of the servo motors.

Dorsal Extension is a change in the arc length due to a change in joint radius, shown in the following equation

$$
E_{i}\left(\emptyset_{n}\right)=R_{n} \emptyset_{n}
$$

Frontal Extension was derived using a geometric, equating to the following equation

$$
\begin{aligned}
& E_{i}\left(\emptyset_{n}\right)=\operatorname{sqrt}\left(A^{2}+B^{2}\right) \\
& \quad A=t_{n}+f_{n} \sin \left(\emptyset_{n}\right)-t_{n+1} \cos \left(\emptyset_{n}\right) \\
& B=f_{n+l} \cos \left(\emptyset_{n}\right)-t_{n+l} \sin \left(\emptyset_{n}\right)+l_{n}
\end{aligned}
$$

\begin{tabular}{cl}
\hline & \multicolumn{1}{c}{ Nomenclature } \\
\hline $\mathrm{t}[\mathrm{mm}]$ & Thickness of link from centerline \\
$\mathrm{f}[\mathrm{mm}]$ & Length from joint center to tendon fixture \\
$\varnothing[\mathrm{rad}]$ & Angle the joint with respect to previous link \\
$1[\mathrm{~mm}]$ & Length of link from joint to joint \\
\hline
\end{tabular}

Each formula represents the tendon's change in length due to a rotational joint. The full extension formula for each tendon is sum of extension formulas of each joint from the base.

\section{$3 \quad$ Results}

The complete endoskeletal structure, shown in Figure 3, was created with a lightweight polyamide polymer. The overall weight of the hand, excluding the actuators, is less than 100 grams.

Preliminary measurements of a single finger was conducted using a high speed camera, pco.edge (pco., Germany). The camera recorded the movements of the finger at 140 frames per second. A checkerboard with (2x2) $\mathrm{mm}$ squares was placed in the background to serve as a measuring reference. Each joint was controlled to move to their maximum flexion angle 5 times to test for repeatability and accuracy. A straight finger was taken as the base reference for angle measurement.

The average time to fully flex a finger was recorded at $0.64 \mathrm{~s}$. This is an average flexion speed of $2.8 \mathrm{rad} / \mathrm{s}$. Although this is only a fraction of the speed capable of the human hand (40 rad/s [9]), this is comparable to current robotic hands and prostheses $[2,6]$.

The visual recording also provided validation for the extension formulas. Each link was observed to have a repeatability of less than a $5^{\circ}$ deviation between each trial. The location of the tip of the finger was observed to maintain a repeatability of within $2 \mathrm{~mm}$. Table 1 summarises the experimental range of motion with the expected values. There was an observed deviation with the theoretical values, however this was expected due to the low resolution of the servo motors. Each motor has a minimum resolution of $5.4^{\circ}$. When attached to the capstans, this resolution is amplified to an error of $17^{\circ}$ in joint movement.

A smaller minimum angle was also observed. During flexion of the DIP and PIP joint, the MCP extended. This is attributed to the inherent coupling of the joints due to a flexible-linked actuation system. When the tendons contract, they will naturally attempt to form the shortest distance between fixture points. Any non-stationary point that come into contact will have a force exerted on it. Subsequently, non-fixed joints will have a moment applied, causing an undesired rotation. Thus, joints further from the base are more prone to joint coupling, in particular the DIP and PIP. Although this effected was slightly mitigated by changing the link geometry, a small

\begin{tabular}{lll}
\hline & Expected & \multicolumn{1}{c}{ Observed } \\
\hline \multirow{2}{*}{ MCP } & Max: 90 & Max:71 \\
& Min: 0 & Min: 0 \\
& Max: 110 & Max: 101 \\
DIP & Min: 0 & Min: $-3^{\circ}$ \\
& Max: 90 & Max: 110 \\
PIP & Min: $0^{\circ}$ & Min -2 \\
\hline
\end{tabular}

Table 1 Observed range of motion of each joint. 


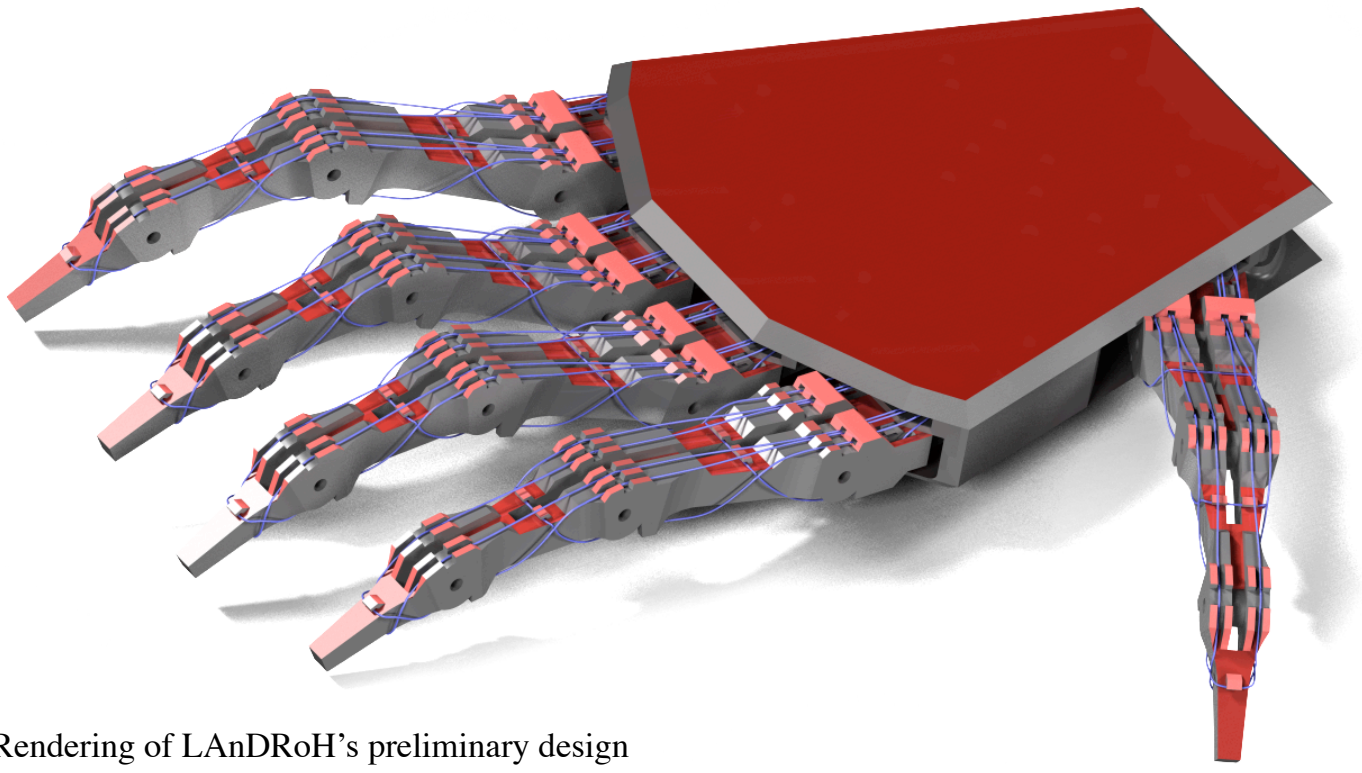

Figure 3 Rendering of LAnDRoH's preliminary design

amount of torque is still being applied causing the undesired effect.

\section{Conclusion}

In this paper, the preliminary concept and design of a lowcost, fully dexterous anthropomorphic hand was presented. It is capable of the same range of motion as the human hand with comparable speeds to current prosthesis. The motions are based on a flexible linkedhosted actuation system that uses braided Dyneema ${ }^{\circledR}$ tendons. The endoskeletal structure was created using a polyamide powder sintered with a rapid prototype machine. The design of the links implemented a hyperbolic curvature to reduce contact points of the tendons with the structure, subsequently reducing the internal friction and joint coupling.

Although the resolution of the joints are not as high as preferred, this work can still serve as a low-cost research tool used for interfacing more complex systems such as brain machine interfaces, or virtual reality systems like haptic control. Further adaptation and refinement of the design can later be used as a lightweight, low-cost prosthetic hand.

Acknowledgements This work has been supported by the mechanical design engineers at the Fraunhofer Institute and the robotics engineers at the Institute of Robotics and Cognitive Systems at the University of Lübeck.

\section{References}

[1] Raichle, Katherine et al.: Prosthesis use in persons with lower-and upper-limb amputation. Journal of Rehabilitation Research and Development. 25(7): pp. 961-972, 2008.
[2] Schulz, S., Pylatiuk, C. and Bretthauer, G., “A New Ultralight Anthropomorphic Hand", Proc. of the 2001 IEEE International Conference on Robotics \& Automation, Seoul, Korea, pp. 2437-2441, 2001.

[3] Kawabuchi, I. "A Designing of Humanoid Robot Hands in Endoskeleton and Exkoskeleton Styles" in Humanoid Robotos: New Development, 2007.

[4] Liu, H. et al., "DLR's multisensory articulated hand, part II: The parallel torque/position control system", Proc. IEEE Int. Conf. on Robotics and Automation, Leuven, Belgium. pp.2087-2093, 1998

[5] Lovchik, C.S. and Diftler, M.A. "The robotnaut hand: The dexterous robot hand for space," Proc. IEEE Int. Conf. on Robotics and Automation, Detroit, MI pp. 907-912, 1999.

[6] Bundhoo, V., Park, E.J., "Design of an artificial muscle actuated finger towrads biomimetic prosthetics", Advanced Robotics, 2005.

[7] Jacobsen, S.C. et al., "Control strategies for tendondriven manipulators", Control Systems Magazine IEEE, 1990

[8] DSM Dyneema, "Dyneema Comprehensive Factsheet”, NC, USA, 2008.

[9] Weir, R.F., "The Design of artificial arms and hands for prosthetic applications" in Biomedical Engineering and Design Handbook, Volume 2. Myer Kutz. McGrawHill, 2009.

[10] Takaki, T. and Omata, T., "High Performance Anthropomorphic Robot Hand with Grasp Force Manification Mechanism", IEEE Int. Conf. on Robotics and Automation, Kobe, Japan. pp. 1691-1703, 2009 